\title{
Dynamical Analysis of HIV/AIDS Epidemic Model with Treatment
}

\author{
Badria Ulfa ${ }^{1}$, Trisilowati $^{2 *}$, Wuryansari Muharini Kusumawinahyu ${ }^{2}$ \\ ${ }^{1}$ Master Program of Mathematics, Faculty of Mathematics and Natural Sciences, University of Brawijaya, Malang, \\ Indonesia \\ ${ }^{2}$ Department of Mathematics, Faculty of Mathematics and Natural Sciences, University of Brawijaya, Malang, Indonesia
}

\begin{abstract}
In this article, an epidemic model of HIV/AIDS with treatment is observed. This model consists of five populations: susceptible, educated susceptible, exposed, HIV infected, and AIDS infected. Antiretroviral therapy (ART) is one type of treatment that can be given to individual that is infected by HIV. This medication can prevent the growth of the virus. Exposed individuals are given short term antiretroviral treatment called Post Exposure Profilaxis (PPP), while for infected individuals are given treatment by combining two or three types of antiretroviral drugs. Dynamical analysis is performed by determining equilibrium points and local stability analysis. Based on the analysis results, two equilibrium points are obtained, namely disease free equilibrium point and endemic equilibrium points. The stability analysis shows that the free equilibrium point is locally asymptotically stable if $R_{0}<1$ and the endemic equilibrium point is locally asymptotically stable under certain conditions. Numerical simulations show that giving two medications together has a greater effect in reducing the spread of the disease.
\end{abstract}

Keywords: antiretroviral, dynamical analysis, HIV/AIDS.

\section{INTRODUCTION}

In medical science, diseases are differentiated into infectious and non infectious diseases. Infectious diseases are diseases caused by bacteria, fungi, viruses, and parasites that can spread through various media. Infectious diseases are a big problem because they can lead to high relative mortality rates, as example is HIV/AIDS.

AIDS (Acquired Immuno Deficiency Syndrome) was first identified in 1981 in Los Angeles, USA. Furthermore, in 1983 has identified the virus that causes AIDS is HIV (Human Immunodeficiency Virus), which is a virus that attacks and damage the human immune system [1]. The spread of HIV virus can occur through several media, such as sexual contact with HIV positive individuals, by sharing needles, by blood transfusions from HIV positive individuals, and through the vertical transmission process, which is mother to child HIV transmission [2].

Treatments that can be done to deal with the spread of HIV/AIDS is the treatment of post exposure prophylaxis (PPP) and antiretroviral (ART). Exposure is an event that causes the risk of transmission. PPP treatment is a short term antiretroviral treatment used to prevent infection

\footnotetext{
* Correspondence address:

Trisilowati

Email : trisilowati@ub.ac.id

Address : Dept. Mathematics, Faculty of Mathematics and Natural Sciences, University of Brawijaya, Veteran Malang, Malang 65145.
}

after a risk incident. This treatment should be given as soon as possible after exposure, which is in the range of two to four hours. It is stated that the provision of PPP after seventy two hours is declared ineffective [3], so it is recommended to provide treatment by combining two or three types of antiretroviral drugs. ARV treatment works by inhibiting the progression of HIV in CD4 ${ }^{+}$T-cells, so it can depress the course of the disease and improve the quality of life [2].

Some mathematical models related to the spread of HIV/AIDS have been studied by some researchers. In 2000, Culshaw [4] discussed a delay time differential equation model of HIV infection of $C D 4^{+}$T-cells. In his study, Culshaw proposed a model for the spread of HIV infection in three compartments, i.e $\mathrm{T}_{v} \mathrm{I}_{x}$ and $\mathrm{V}$.

In 2009, Cai [5] analyzed the model and stability of the HIV/AIDS epidemic with treatment. The process of transmission of the disease occurs due to the presence of susceptible individuals who interact with individuals exposed and infected with HIV. In this model, the HIV/AIDS epidemic is divided into four compartments, that is SIJA. Each compartment states the subpopulation of susceptible individuals $(S)$, exposed $(I)$, infected $(D)$, and AIDS infectious (A).

In 2014, Mahato [6] analyzed the mathematical model of HIV/AIDS with vertical transmission. In this study, the disease epidemic model are expressed in four compartments, i.e $S_{x} E_{x} I_{x} A$, with the spread of the disease affected by verti- 
cal transmission, that is transmission from pregnant mother to the baby.

Furthermore, in 2015, Basak [7] proposed a model of the HIV/AIDS epidemic which is expressed in four compartments, namely $S_{x} I_{1}, I_{2} x A$. The process of spreading of the disease is assumed to occur due to the interaction between susceptible individuals with exposed and infected individuals.

In 2016, Huo [8], examined the epidemic model of HIV/AIDS by providing treatment for infected individuals. Huo divided the compartment model of HIV/AIDS epidemic into five parts, namely subpopulation of susceptible individuals $(S)$, infected $(I)$, AIDS infectious $(A)$, therapy recipients $(T)$, and subpopulations for individuals who change sexual habits $(R)$. In this model, HIV transmission occurs when there are susceptible individuals who interact with HIV infected individuals, thus forming a subpopulation of $I$ without exposed compartment.

In 2016, Marsudi [9], analyzed the sensitivity of the HIV virus transmission model with the effect of screening and therapy. The disease model distribution compartment is divided into six, that is susceptible $(S)$, educated susceptible $(E)$, unaware infectives $\left(I_{1}\right)$, aware infectives $\left(I_{2}\right)$, therapy infectives $(T)$, and AIDS infectious $(A)$. The spread of the virus is declared to occur due to the interaction between susceptible individuals with individual unaware infectives, aware infectives, and therapy infectives [9].

Different from previous research, this article examines the epidemic model of HIV/AIDS disease transmission with treatment based on modified results from previous studies of Huo [8] and Marsudi [9]. Modification is done by assuming the disease transmission occurs because of the interaction between susceptible individuals and infected individuals [8]. In addition, treatment is given directly to exposed and infected individuals without forming a subpopulation of individual therapy. In this paper, dynamical analysis is performed by determining the equilibrium points and analyzing their local stability. Finally, numerical simulations are performed to verify the analytical results.

\section{MATERIALS AND METHODS Contruction Model}

In this article, the epidemic model of HIV/AIDS with treatment is expressed in five compartments. Each compartment describes the process of spreading the disease from one compartment to another compartment that is affected by time $(t)$. In this study, the HIV/AIDS epidemic model is in the form of a nonlinear autonomous system.

\section{Determination of the Equilibrium Point}

In dynamical analysis, the first step is determining the equilibrium point of the model. The equilibrium point can be done by solving nullcline. In general, the equilibrium point of the disease distribution model is divided into two types, namely the point of disease free equilibrium and endemic equilibrium, with each point representing whether or not there is a disease in a population.

\section{Stability of the Equilibrium Point}

Stability analysis is done by linearizing the system of equations to form a Jacobian matrix. Linearization is done with purpose to transform the nonlinear system into linear, so the stability of the equilibrium points can be observed easily. Furthermore, based on the Jacobian matrix, the eigenvalue of the characteristic equation can be determined. If all eigenvalue are negative then the equilibrium point is locally asymptotically stable. On the other hand, it is unstable if at least there is one negative eigenvalue.

\section{Numerical Simulation}

Numerical simulations was performed to verify the analytic results and illustrate the behavior of the model. This step is done by using fourth order Runge Kutta method with MATLAB software. The parameter values should be choosen in order that can support the calculation result analytically.

\section{RESULT AND DISCUSSION Model Formulation}

The epidemic model of HIV/AIDS with treatment is obtained based on modified studies [8] and [9]. Modification is done on the process of the spread occurrence of the illness which is assumed as in the previous study [8]. The treatment is given directly to subpopulation of exposed and infected individuals with the aim of reducing the spread of the disease.

The model consists of five subpopulations: subpopulation of susceptible individuals $(S)_{x}$ educated susceptible $(E)_{x}$ exposed $\left(I_{1}\right)_{x}$ HIV infected $\left(I_{2}\right)$, and AIDS infected $(A)$. Each population $S E I_{1} I_{2} A$ is positive because it states population density. The compartment diagram of the epidemic model $S E I_{1} I_{2} A$ with treatment is described in Figure 1.

The rate of change in the number of individuals susceptible subpopulation (S) is influenced by 
several conditions, namely the birth rate denoted by $A$, educational programs for susceptible individuals at rate $\eta$, and natural death at rate $\mu$. In addition, when susceptible individuals come in contact with HIV infected individuals, they become infected at rate $\beta_{1}$ and then move to the exposed compartment.

In educated susceptible subpopulation which is denoted by $(E)$, the rate of change individuals number is affected by the presence of educated susceptible individuals at rate $\eta$, natural death at rate $\mu$ and the rate of exposed individuals become HIV infected individuals because of the effect of interacting with HIV infected individuals at rate $\beta_{2}$. Furthermore, this subpopulation changes in the number of individuals are also affected by the presence of exposed individuals become susceptible individual again after receiving PPP treatment at rate $\mathrm{u}_{1}$.

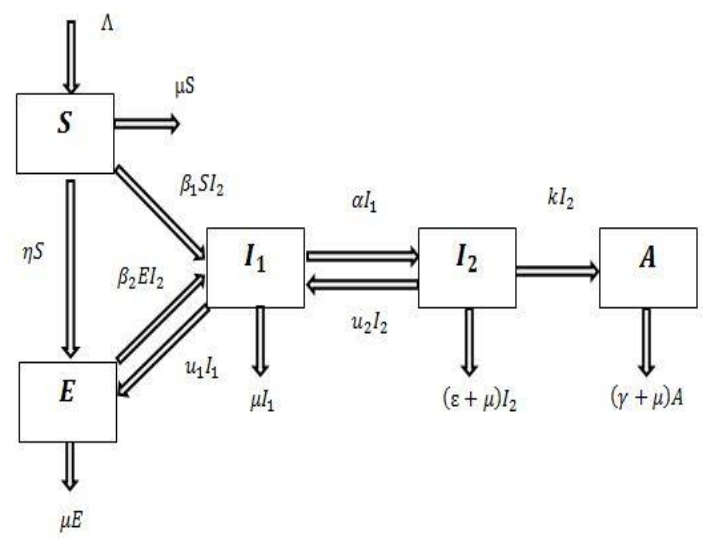

Figure 1. Compartment diagram of HIV/AIDS epidemic model with treatment.

The number of exposed subpopulation individuals $\left(I_{1}\right)$ is increased due to effective contact between susceptible individuals $(S)$ and HIV infected individuals $\left(I_{2}\right)$ at rate $\beta_{1} S$ as well as due to effective contact between educated susceptible individuals $(E)$ and HIV infected individuals $\left(I_{2}\right)$ at rate $\beta_{2} E$. Besides that, this compartment is influenced by the rate of exposed individuals become HIV infected individuals at rate $\alpha$, natural death at rate $\mu$, PPP treatment for exposed individuals at rate $u_{1}$, and there are HIV infected individuals which become exposed individual again after getting antiretroviral treatment (ART) at rate $\mathrm{u}_{2^{*}}$

HIV infected subpopulation $\left(I_{2}\right)$ is affected by parameters: $\alpha, k, \mu$, and $\varepsilon$. Parameter $\alpha$ represents HIV transmission rate, $k$ is the transmission rate from HIV infected subpopulation to AIDS infected subpopulation.
Furthermore, there is an antiretroviral treatment (ART) for HIV infected individuals at rate $\mathrm{u}_{2}$.

Finally, the change rate of AIDS subpopulation (A) is influenced by several parameters, such as, $k$ and $\mu$ defined as before and $\gamma$ denotes the death rate due to AIDS infection.

Based on the description above, it is obtained the following differential equation system

$$
\begin{aligned}
& \frac{d S}{d t}=\Lambda-\beta_{1} S I_{2}-(\eta+\mu) S_{2} \\
& \frac{d E}{d t}=\eta S-\beta_{2} E I_{2}-\mu E+u_{1} I_{1} \\
& \frac{d I_{1}}{d t}=\beta_{1} S I_{2}+\beta_{2} E I_{2}-\left(\alpha+\mu+u_{1}\right) I_{1}+u_{2} I_{2} \\
& \frac{d I_{2}}{d t}=\alpha I_{1}-\left(k+\varepsilon+\mu+u_{2}\right) I_{2} \\
& \frac{d A}{d t}=k I_{2}-(\mu+\gamma) A_{x}
\end{aligned}
$$

with $\beta_{2}<\beta_{1}$.

\section{Equilibrium Point of Model}

In dynamical analysis, determining the equilibrium point of the epidemic model $S E I_{1} I_{2} A$ is obtained by solving the equation $\frac{d S}{d t}=\frac{d E}{d t}=\frac{d I_{1}}{d t}=\frac{d I_{2}}{d t}=\frac{d A}{d t}=0$. Based on the system (1), it is obtained the disease free equilibrium point

$$
X_{0}=\left(S_{0}, E_{0}, I_{10}, I_{20}, A_{0}\right)=\left(\frac{A}{\eta+\mu}, \frac{\eta \Lambda}{\mu(\eta+\mu)}, 0,0,0\right) .
$$

The equilibrium point $X_{0}$ means that there is no infected individual of HIV/AIDS in a population, so that the subpopulation of infected individuals, that is $\left(I_{1^{x}} I_{2^{x}} A\right)$ are zero. Endemic equilibrium point is stated as

$$
\begin{aligned}
& X^{*}=\left(S^{*}{ }_{s} E^{*}{ }_{n} I_{1}{ }^{*} I_{2}{ }^{*} A^{*}\right) \text { with } \\
& S^{*}=\frac{\beta_{1} I_{2}^{*}+\eta+\mu^{*}}{E^{*}}=\left(\frac{\eta \Lambda \alpha+u_{1} I_{2}^{*}\left(k+\varepsilon+\mu+u_{2}\right)\left(\beta_{1} I_{2}^{*}+\eta+\mu\right)}{\left(\beta_{1} I_{2}^{*}+\eta+\mu\right)\left(\beta_{2} I_{2}^{*}+\mu\right) \alpha}\right), \\
& I_{1}^{*}=\frac{\left(k+\varepsilon+\mu+u_{2}\right) I_{2}^{*}}{\alpha}, \\
& A^{*}=\frac{k I_{2}^{*}}{(\mu+\gamma)} .
\end{aligned}
$$

and the equilibrium point $I_{2}^{*}$ is obtained by solving the third degree polynomial equation as follows.

$I_{2}^{3} K+I_{2}^{2} L+I_{2} M=0$.

Equation (2) is equivalent to 


$$
I_{2}=0 \quad \text { or } \quad I_{2}^{2} K+I_{2} L+M=0 .
$$

with

$$
\begin{aligned}
K= & \left(\alpha k \beta_{1} \beta_{2}+\alpha \varepsilon \beta_{1} \beta_{2}+\alpha \mu \beta_{1} \beta_{2}+\mu k \beta_{1} \beta_{2}\right. \\
& \left.+\mu \varepsilon \beta_{1} \beta_{2}+\mu^{2} \beta_{1} \beta_{2}+\mu u_{2} \beta_{1} \beta_{2}\right)_{x} \\
L= & \left(\alpha k \beta_{1} \mu+\alpha k \beta_{2} \eta+\alpha k \beta_{2} \mu+\alpha \varepsilon \beta_{1} \mu+\alpha \varepsilon \beta_{2} \eta\right. \\
& +\alpha \varepsilon \beta_{2} \mu+\alpha \mu^{2} \beta_{1}+\alpha \mu \beta_{2} \eta+\alpha \mu^{2} \beta_{2}+\mu^{2} k \beta_{1} \\
& +\mu k \beta_{2} \eta+\mu^{2} k \beta_{2}+\mu^{2} \varepsilon \beta_{1}+\mu \varepsilon \beta_{2} \eta+\mu^{2} \varepsilon \beta_{2} \\
& +\mu^{2} \beta_{1}+\mu^{2} \beta_{2} \eta+\mu^{2} \beta_{2}+\mu^{2} u_{2} \beta_{1}+\mu u_{2} \beta_{2} \eta \\
& +\mu^{2} u_{2} \beta_{2}+u_{1} k \beta_{1} \mu+u_{1} \varepsilon \beta_{1} \mu+u_{1} \mu^{2} \beta_{1} \\
& \left.\quad+u_{1} u_{2} \beta_{1} \mu-\beta_{1} \beta_{2} \Lambda \alpha\right)_{x} \\
M= & \left(\alpha k \eta \mu+\alpha k \mu^{2}+\alpha \varepsilon \eta \mu+\alpha \varepsilon \mu^{2}+\alpha \mu^{2} \eta+\alpha \mu^{2}\right. \\
& +\mu^{2} k \eta+\mu^{3} k+\mu^{2} \varepsilon \eta+\mu^{2} \varepsilon+\mu^{3} \eta+\mu^{4}+\mu^{2} u_{2} \eta \\
& +\mu^{2} u_{2}+u_{1} k \eta \mu+u_{1} k \mu^{2}+u_{1} \varepsilon \eta \mu+u_{1} \varepsilon \mu^{2} \\
& +u_{1} \mu^{2} \eta+u_{1} \mu^{3}+u_{1} u_{2} \eta \mu+u_{1} u_{2} \mu^{2}-\beta_{1} A \mu \alpha \\
& \left.-\beta_{2} \eta \Lambda \alpha\right) .
\end{aligned}
$$

To simplify the solution step, first simplified equation (3) becomes

$I_{2}^{2}+I_{2} \frac{L}{K}+\frac{M}{K}=0$,

which can be written in form

$I_{2}^{2}+I_{2} Q+R=0$,

with

$$
Q=\frac{L}{K^{\prime}} \quad \text { and } \quad R=\frac{M}{K^{*}}
$$

Based on equation (4) we obtained several possible equilibrium points $I_{2}^{*}$ as follows.

In order to obtain an equilibrium point $I_{2}^{*}$ a positive real number, it must satisfy

$Q^{2}-4 R \geq 0$,

and the solution is divided into four cases:

1. $R>0$ and $Q<0$,

i. If $Q^{2}=4 R$, then there are two positive twin roots, that is

$I_{2_{ \pm}}=\frac{-Q}{2}$

ii. If $Q^{2}>4 R_{x}$ then there are two dif-

ferent positive roots, that is

$I_{2_{ \pm}}=\frac{-Q \pm \sqrt{Q^{2}-4 R}}{2}$.

2. If $R<0$ and $Q<0$, then one positive root is obtained, that is

$$
l_{2}=\frac{-Q+\sqrt{Q^{2}-4 R}}{2} \text {. }
$$

3. If $R=0$, and $Q<0$, then obtained two positive roots, that is

$$
I_{2}=-Q
$$

4. If $R<0$, and $Q>0$, then one positive root is obtained, that is

$$
I_{2}=\frac{-Q+\sqrt{Q^{2}-4 R}}{2} \text {. }
$$

The equilibrium point $X^{*}$ states that $\left(I_{1}, I_{2}, A\right)$ exsist in the population. This condition represents that in the population epidemic of HIV/AIDS disease will occur.

\section{Stability of the Equilibrium Point}

The stability analysis of the equilibrium point is investigated by linearizing of system (1). From this linearization, it is obtained the following Jacobian matrix

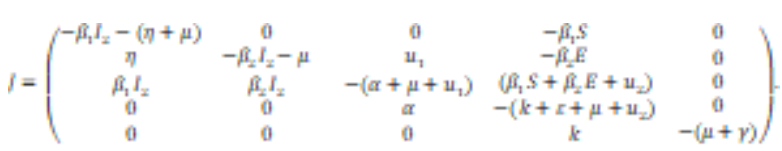

The Jacobian matrix of the disease free and endemic equilibrium points is obtained by substituting each equilibrium point in the Jacobian matrix.

Teorema 1. The disease free equilibrium point $\left(X_{0}\right)$ of epidemic model $S E I_{1} I_{2} A$ is locally asymptotically stable if $R_{0}<1$.

Proof. If it is given a disease free equilibrium point

$X_{0}=\left(S_{0}, E_{0}, I_{10}, I_{20}, A_{0}\right)=\left(\frac{\Lambda}{\eta+\mu}, \frac{\eta \Lambda}{\mu(\eta \eta+\mu \nu}, 0,0,0\right)$, then the Jacobian matrix $X_{0}$ is as follows

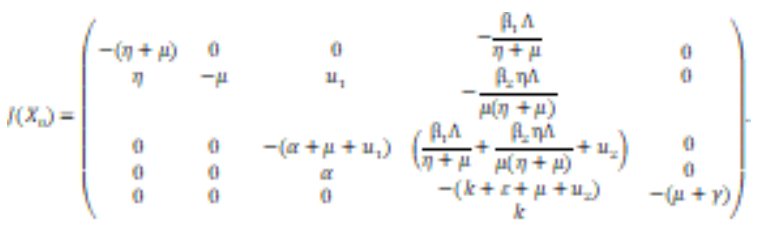

The eigenvalues of the $l\left(X_{0}\right)$ matrix are obtained by solving the characteristic equation

$\left|J\left(x_{0}\right)-\lambda I\right|=0$.

The eigenvalues of the above matrix are $\lambda_{1}=-(\eta+\mu), \lambda_{2}=-\mu, \lambda_{2}=-(\mu+\gamma)$.

whereas for $\lambda_{4}$ and $\lambda_{5}$ are obtained by determining trace value and determinant of the submatrix

$l_{1}\left(X_{0}\right)=\left(\begin{array}{cc}-\left(\alpha+\mu+u_{1}\right) & \left(\frac{\beta_{1} \Lambda}{\eta+\mu}+\frac{\beta_{2} \eta \Lambda}{\mu(\eta+\mu)}+u_{2}\right) \\ \alpha & -\left(k+\varepsilon+\mu+u_{2}\right)\end{array}\right)$,
trace $J_{1}\left(X_{0}\right)=-\left(\alpha+2 \mu+u_{1}+k+\varepsilon+u_{2}\right)<0$,

and 
(Ulfa et al.)

$$
\begin{gathered}
\operatorname{det} J_{1}\left(X_{0}\right)=\left(\alpha+\mu+u_{1}\right)\left(k+\varepsilon+\mu+u_{2}\right) \\
-(\alpha)\left(\frac{\beta_{1} \Lambda}{\eta+\mu}+\frac{\beta_{2} \eta \Lambda}{\mu(\eta+\mu)}+u_{2}\right) \\
\left(\alpha+\mu+u_{1}\right)\left(k+\varepsilon+\mu+u_{2}\right)-(\alpha)\left(\frac{\beta_{1} \Lambda}{\eta+\mu}+\frac{\beta_{2} \eta \Lambda}{\mu(\eta+\mu)}+u_{2}\right)>0 \\
\left(\alpha+\mu+u_{1}\right)\left(k+\varepsilon+\mu+u_{2}\right)>(\alpha)\left(\frac{\beta_{1} \Lambda}{\eta+\mu}+\frac{\beta_{2} \eta \Lambda}{\mu(\eta+\mu)}+u_{2}\right) \\
1>\left(\frac{a \mu\left(\beta_{2} \Lambda+\alpha \beta_{2} \eta \Lambda+a u_{2} \mu(\eta+\mu)\right.}{\mu(\eta+\mu)\left(\alpha+\mu+u_{1}\right)\left(k+\varepsilon+\mu+u_{2}\right)}\right)
\end{gathered}
$$

Suppose

$\left(\frac{\alpha \mu \beta_{2} \Lambda+\alpha \beta_{2} \eta \Lambda+\alpha u_{\mu} \mu(\eta+\mu)}{\mu(\eta+\mu)\left(\alpha+\mu+u_{1}\right)\left(k+\varepsilon+\mu+u_{2}\right)}\right)=R_{0}$

then

$R_{0}<1$.

Based on above description, if $R_{0}<1$ then all eigenvalues are negative. It indicates that the disease free equilibrium point is locally asymptotically stable.

Teorema 2. The endemic equilibrium point $\left(X^{3}\right)$ of epidemic model $S E I_{1} I_{2} A$ is locally asymptotically stable if and only if $a_{n}>0, \forall \forall_{n}=0,1,2,3$ and $a_{1} a_{2}-a_{0} a_{a}>0$

Proof. If an endemic equilibrium point is given $X^{*}=\left(S^{*}{ }_{v} E^{*}{ }_{v} I_{1}{ }^{*} I_{2}{ }^{*}{ }^{*} A^{*}\right)$, then the Jacobian $f\left(X^{*}\right)$ matrix is obtained as follows

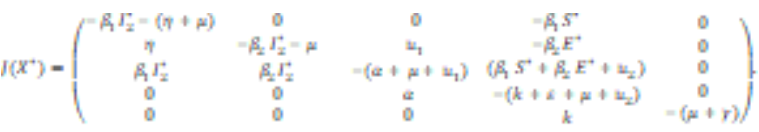

The eigenvalues of the $f\left(X^{*}\right)$ matrix are obtained by solving the equation

$\left|j\left(X^{*}\right)-\lambda I\right|=0$.

The eigenvalues of this matrix are $\lambda_{1}=-(\mu+\gamma), \lambda_{2}=-\left(\beta_{2} I_{2}^{*}+\mu\right)$, while for $\lambda_{3}, \lambda_{4}$ and $\lambda_{5}$ are obtained based on the solution of a third degree polynomial which is easier solve by using Routh Hurwitz criterion on [10].

The general form of a polynomial equation of third degree is

$a_{0} \lambda^{3}+a_{1} \lambda^{2}+a_{2} \lambda+a_{3}=0$.

By using Routh Hurwitz criterion, from equation (5) the root of the real part is negative if and only if $a_{n}>0, \forall_{n}=0,1,2,3$ and $a_{1} a_{2}-a_{0} a_{a}>0$. Thus, the endemic equilibrium point is locally asymptotically stable.

\section{Numerical Method and Simulations}

In this section, the simulation results show the change of the number of individuals in each subpopulation $S_{x} E_{x} I_{1 x} I_{2 x} A$ against time. Numerical simulation is executed using MATLAB software which are presented in the following figures (see Fig. 2, Fig. 3, Fig. 4a, Fig. 4b and 4c).

Figure 2 is conducted by using the following parameter values,

$A=10, \beta_{1}=0.00005, \beta_{2}=0.0000001, \eta=$

$0.003, \mu=0.01, \alpha=0.2, k=0.01, \varepsilon=$

$0.05, \gamma=0.02$

$u_{1}=0, u_{2}=0$. Based on these parameter values, it is obtained $R_{0}=0.5236<1$. The graph is shown with two different initial values,

$(250,200,100,100,10)$ and

$(400,220,180,120,85)$ with each solution convergent toward the point

$(769.23,230.77,0,0,0)$. The solution behavior of the subpopulation $S$ convergent toward point 769.23 and $E$ converget to point 230.77 , while subpopulation $I 1, I 2, A$ tend to zero. This means, that all solutions move toward the point of disease free equilibrium $\left(X_{0}\right)$ and the numerical simulations obtained are consistent with the results of the analysis that $X_{0}$ is locally asymptotically stable. This condition indicates that there is no transmission of disease in the population.
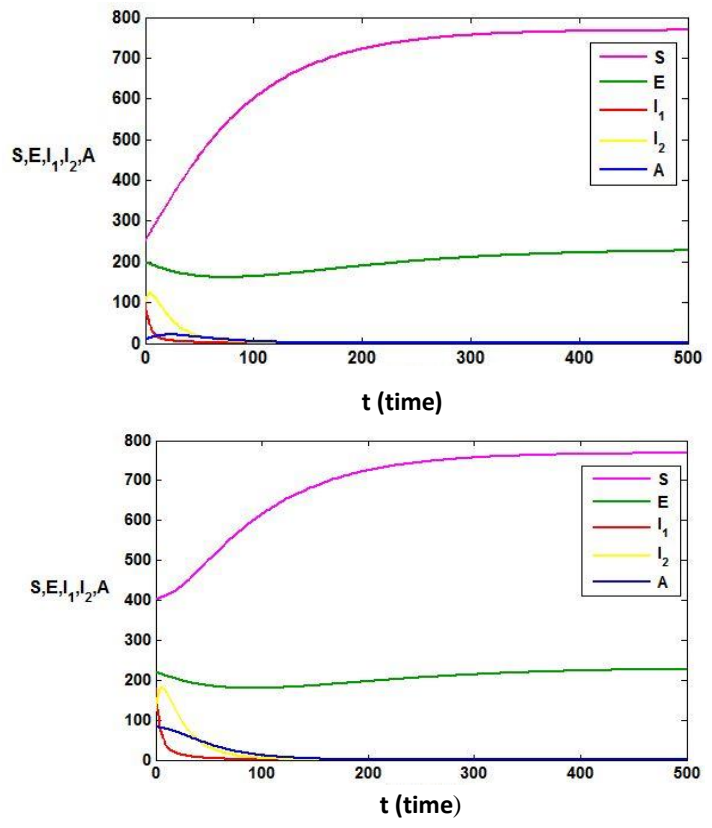

Figure 2. The solution of epidemic model $S E I_{1} I_{2} A$ at the equilibrium point $X_{0}$.

Figure 3 is illustrated to show the stability of the endemic equilibrium. Parameter values 
$A=100, \beta_{1}=0.0001, \beta_{2}=0.00005, \eta=$

$0.003, \mu=0.01, \alpha=0.5, k=0.05, \varepsilon=$

$0.05, y=0.02, u_{1}=0.03, u_{2}=0.02$

are used in this simulation. These parameters satisfy case two with $R=-1.764618117 \times 10^{5}$ and $Q=-544.2451155$, which implies the endemic will occur in the population. Moreover, it is obtained

$a_{2}=0.06054611822, a_{a}=0.004265827497 x$ and $a_{1} a_{2}-a_{0} a_{a}=0.04183534505$.

By Theorem 2, this indicates that the endemic equilibrium point is locally asimtotically stable which is supported by numerical simulation (Fig. 3). The initial value used in this simulations are

$(250,175,150,75,50)$ and $(400,220,180,120,85)$,

it can be seen that all solution convergent to the point

$(1107.87,192.26,200.89,772.64,1287.73)$.

Based on these initial condition, the exposed and infected subpopulation convergent toward point 200.89 and 772.64 respectively as time tends to infinity. This condition implies that the disease remains in the population.
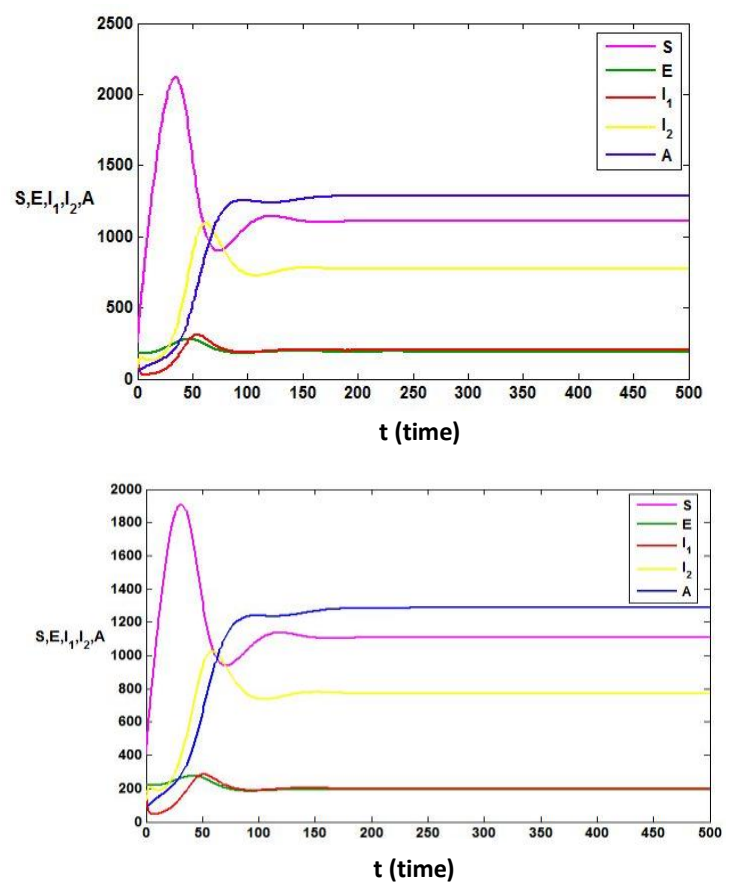

Figure 3. The solution of $S E I_{1} I_{2} A \mathrm{f}$ at the endemic equilibrium $X^{*}$ when $R<0$ and $Q<0$.

In the HIV/AIDS epidemic model, it is introduced the parameters $u_{1}$ and $u_{2}$ representing PPP and antiretroviral treatment respectively which is used to help reducing the number of individuals exposed and HIV infected. Figure 4 (a, $b, c)$ states that the changes in parameter values of $u_{1}$ and $u_{2}$ give a significant effect on the number of HIV infected individuals $\left(I_{2}\right)$.

This is indicated by increasing the values of $u_{1}$ and $u_{2}$ in the model will result in decreasing the number of infected population. Even the infected population tends to zero along with the increasing the values $u_{1}$ and $u_{2}$ simultaneously as indicated in Figure 4c. The changing of the value $u_{1}$ and $u_{2}$ is given in the legend of the figure. Figure $4 \mathrm{c}$ also shows that by applying the PPP treatment $\left(u_{1}\right)$ and ARV treatment $\left(u_{2}\right)$ together give a greater effect than applying PPP treatment only on Figure $4 a$ or ARV treatment only on Figure $4 b$. Therefore, to reduce the number of individuals $I_{2}$ is better if both treatments are applied together in order to obtain optimal results.
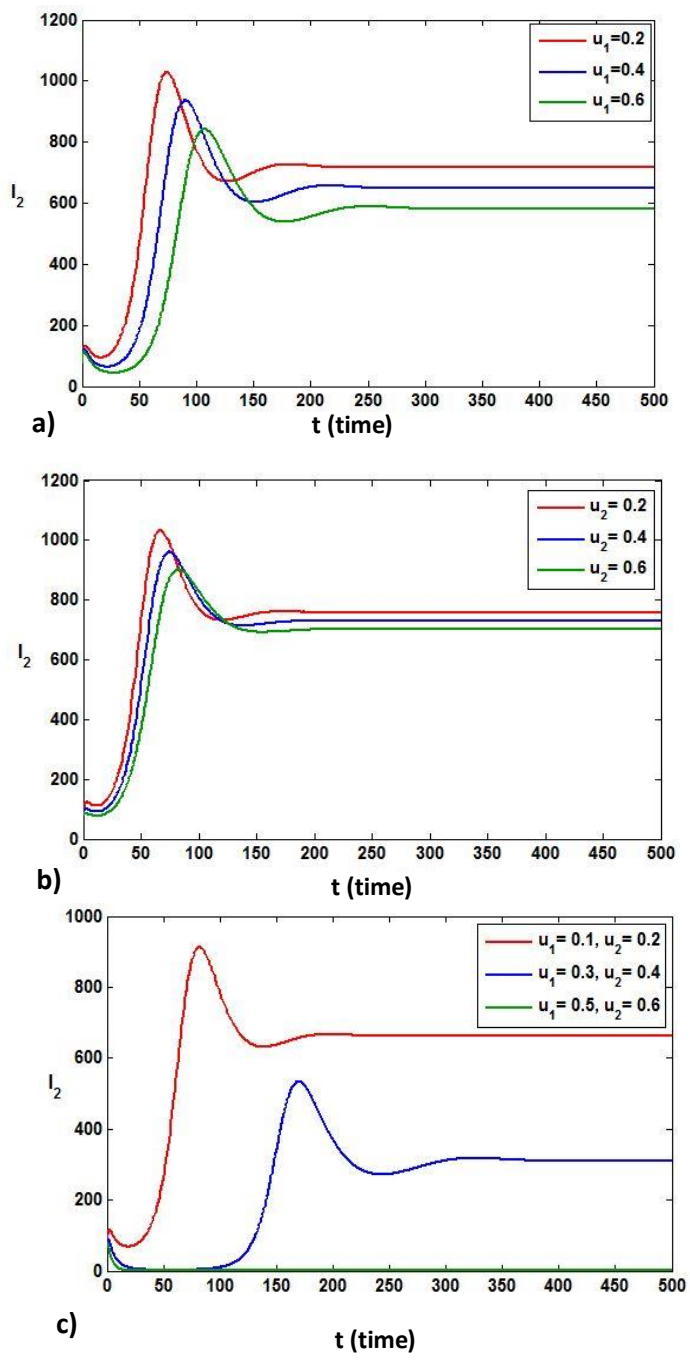

Figure 4. The Effect of Changing Values. a) $u_{1}$ on the subpopulation $I_{2}$, b) $u_{2}$ on the subpopulation $I_{2}$, and c) $u_{1}$ and $u_{2}$ on the subpopulation $I_{2}$ 


\section{CONCLUSION}

In this study, a HIV/AIDS epidemic model has been observed. The analysis results show that there are two equilibrium points of the model, namely the disease free equilibrium point which is locally asymptotically stable if $R_{0}<0$ and an endemic equilibrium point which is locally asymptotically stable under certain conditions.

The numerical simulation performed support the the analytical result that has been obtained at the result and discussion section, so that both free disease and endemic solutions provide the appropriate results. In addition, the presence of antiretroviral treatment denoted by $u_{1}$ and $u_{2}$ indicates that PPP and ARV treatment can be used to assist in reducing the number of HIV infected.

\section{REFERENCES}

[1] UNAIDS and WHO. 2003. A history of the HIV/AIDS epidemic with emphasis on Africa. Department of Economic and Social Affairs United Nations Secretariat. New York.

[2] UNESCO. 2012. Sexually transmitted infections and HIV/AIDS: supplementary guidebook for reproductive health technicians. UNESCO Indonesia. Jakarta.

[3] Indonesian Department of Health. 2007. Antiretroviral therapy: guidelines for clinical management of HIV infection in adults and adolescents. Indonesian Department of Health. Jakarta.

[4] Culshaw, R.V., S. Ruan. 2000. A delay differential equation model of HIV infection of [CD4】^+ T-cells. Math. Biosci. 165. 2739.

[5] Cai, L., X. Li, M. Ghosh, B. Guo. 2009. Stability analysis of an HIV/AIDS epidemic model with treatment. Comput. Appl. Math. 229. 313-323.

[6] Mahato, B., B.K. Mishra., A. Jayswal, R. Chandra. 2014. A mathematical model on Acquired Immunodeficiency Syndrome. The Egypt. Math. Soc. 22. 544-549.

[7] Basak, U.S., B.K. Datta, P.K. Ghose. 2015. Mathematical analysis of an HIV/AIDS epidemic model. Am. J. Math. Stat. 5(5). 253-258.

[8] Huo, H.F., R. Chen, X.Y. Wang. 2016. Modelling and stability of HIV/AIDS epidemic model with treatment. Appl. Math. Model. 40. 6550-6559.

[9] Marsudi, R.B.E. Wibowo, N. Hidayat. 2016. A sensitivity analysis of the impact of educational campaign, screening and therapy on the spread of HIV infection. Nonlinear Anal. Differential Equations. 4. 327-341.

[10] Murray, J.D. 2002. Mathematical biology I: an introduction, $3^{\text {rd }}$ Ed. Springer Verlag. Berlin Heidelberg. 